\title{
Dental Self Treatment--Universal Design: To Prevent Caries and Extractions (Updated 2018)
}

\author{
Samuel A. Nigro, MD* \\ Retired psychiatrist, formerly Assistant Clinical Professor of Psychiatry, Case Western Reserve University \\ School of Medicine \\ *Corresponding Author: Samuel A. Nigro, Retired psychiatrist, formerly Assistant Clinical Professor of \\ Psychiatry, Case Western Reserve University School of Medicine, USA Email: sam@docnigro.com
}

Utility Patent Pending: Dental Self Treatment: Toothpaste with antibiotics, steroids, stannous fluoride supplements will prevent and treat many non-cosmetic dental problems--and may improve body microbiome benefits. Small amount of table salt by finger massage of tooth will help also.

Originally, this was a single self-report of self treatment of severe dental pain and likely enamel deterioration which is fairly common and usually ends up being treated by root canal procedures and/or extractions. Having witnessed many others go through this, I was not happy having the same problem knowing the usual outcome. It has been over two years of "cure", long enough to want to share with others.

Having had all "wisdom" teeth removed as an adolescent, I became a faithful toothbrush and dental floss user with few problems for decades. But at 78 years old, I awoke with throbbing pain at my left lower second molar. It was sensitive to touch, distracting continuously with its discomfort, and totally preoccupying with the occasional sharp pains. The dentist found evidence of low grade infection and prescribed vigorous brushing/flossing, over-the counter mouth washes, ten days of antibiotics and samples of higher fluoride toothpaste. $\mathrm{He}$ referred me to dentists who do extractions whom I did not contact, preferring to avoid such as long as possible. I did not like the toothpaste at all so I just put some on my finger and massaged the tooth and contiguous gum area with it three times a day. I still did my usual brushing with my usual toothpaste early morning and before bedtime.

The symptoms were partially resolved during the first ten days, but not enough and I was about to call the extraction dentists, but hesitated, remembering all my wife had to go through with this four times (four extractions) all ready.

Suddenly I remembered what I did as a physician on the first nuclear missile submarine, the USSGEORGEWASHINGTONSSBN598 (Admiral Rickover wanted "MDs" on board to prevent medical reasons for loss of our 90day submerged war deterrent patrols). Several "toothaches" afflicted a couple of the men on my patrols. I did not want to extract, so I decided that the mouth was "exterior" to the body and topical antibiotics might work. I gave some to the men having them rub the tooth and gum area three times a day. All felt better and we went to twice a day after two weeks and then once a day until patrol was over. The guys dental problems resolved and had not further problems.

Back to my tooth. After doing the oral antibiotics and vigorous mouth cleaning, I had the idea that systemic antibiotic treatment could not reach or sustain adequate levels for toothgum contiguous areas. And I remembered what I did on Navy patrols. So I pulled an old tube of triple antibiotic ointment from my medicine cabinet and began to massage the troubled tooth-gum area with a finger dab (1/2 inch) of the triple antibiotic ointment. This I did three times a day. It was a good massage of about 1-2 minutes each time. I could tell the difference and improvement within several days. I continued it about two weeks with occasional use whenever any increased awareness of the tooth occurred, but all was asymptomatic, to my great relief, for the most part. There was a low grade sense of inflammation so I added topical cortisone $(1 / 2$ inch dab) to the topical antibiotic once a day for a week. I continued twice a day 
for a month and then daily for a month, and all is fine six months later. At the first sign of any dental problem, I rub the topical antibiotic on the area once a day for several days. All has been well for over a year. Then my wife had another dental inflammation which had led to four extractions before. She did my topical antibiotic treatment and was cured. When she went for her routine annual dental care, she said nothing thinking the dentist would find something, but the dentist said all was well! She was very pleased also because this was probably the first time I ever told her anything correct in over fifty years of marriage. A son in law's tooth complaint was also cured.

I have had successful outcomes with several others since including an old male who had three root canals but did not need a fourth after doing the antibiotic massage. Studies show that simple dental cavities are due to infection-occasional antibiotic massage may be preventive of most dental problems.

Then, two years later, my original treated tooth began to throb and hurt again. I started the topical massage again with poor response. I added topical hydrocortisone with some improvement, but after two weeks felt was not enough. I thought I would need to go to the dentist who would likely recommend extraction. So I shifted to dabs of metronidazole and in three days, the tooth was better but still tender. I began then to focus my vibrating toothbrush on the tender tooth using higher fluoride toothpaste. I vibrated specifically on the tender tooth at least thirty seconds each time and followed with the metronidazole. The tooth cleared up in ten days.

Also, dental antibiotic massage might help reduce infections if on steroids for any reason. I was briefly put on steroids for traumatic arthritis and could feel an increase in dental sensitivity, so I started my topical antibiotic dental massage until off the steroids. This makes one consider the "preventive" aspects of topical antibiotic dental massage for infections if in a hospital or wherever there are high concentrations of infectuous disease agents--or a dab into each nostril and on ones gums at the first sign of a cold might help defense against full cold syndrome.

I have discerned no negative aspects to what I have done and hopefully there will be none. Attention should be given to allergies to antibiotics. Certainly, all should be informed if any thing negative occurs or has occurred when (if?) someone did this before. It is hoped that my findings can be duplicated with home-cure relief for all. At this time, I think it should be promulgated as part of routine self-care recommendations.

Review of dental literature reveals that dental caries have been known for decades to be bacteria-caused. The mouth is an "exterior" part of the body. Topical antibiotics and "skin treatments" like metronidazole might be considered for all dental problems?

Back to saving teeth process summary: Use vibrating toothbrush with higher fluoride toothpaste with toothbrush dabbed with antibiotics and steroids massaging vigorously the tender tooth for 30 seconds at least each time. Dab triple antibiotic ointment (or better, I found, a dab of metronidazole cream) and massage into tender tooth four times a day. Dab a pea-size glob of plain table salt onto the hurting tooth--occasionally add a little dab of hydrocortisone ointment and/or triple antibiotic ointment. Nothing is a quick fix--dental recovery is slow--weeks. Nothing guaranteed but worth a try. If not work, see you local professional dentist (Stay away from corporate dental businesses which create and reciprocate expensive "cardiac surgery" scams for your mouth).

YEARS LATER, I conclude that many, if not most, dental problems can be prevented by periodic (weekly?) dental/gum massage with triple antibiotic ointment, steroid ointment, and stannous fluoride supplements. Also suggested was twice weekly saltwater mouth rinsing, which should reduce the bacterial causes of all dental problems. This is especially true for those over 40 years old when "aging" begins to weaken bone-teeth-gum togetherness such that bacteria begin to do their damage. Dentists will have reasons not to do this because they are a "find expensive work to do" pseudo-profession. They will find reasons to do root canals on every one of your teeth. Prevention? Forget about it from dentists.

Dental hygiene may have importance in mental health and healthy brains. The growing research on microbiomes lends credibility that bacteria pervade the human body in many ways and must be linked to oral flora.

\section{How bacteria are changing your mood}

By James Gallagher Presenter, The Second Genome, BBC Radio 424 April 2018 
If anything makes us human it's our minds, thoughts and emotions.

And yet a controversial new concept is emerging that claims gut bacteria are an invisible hand altering our brains.

Science is piecing together how the trillions of microbes that live on and in all of us - our microbiome - affect our physical health.

But even conditions including depression, autism and neurodegenerative disease are now being linked to these tiny creatures.

We've known for centuries that how we feel affects our gut - just think what happens before an exam or a job interview - but now it is being seen as a two-way street.

Groups of researchers believe they are on the cusp of a revolution that uses "mood microbes" or "psychobiotics" to improve mental health.

The study that ignited the whole concept took place at Kyushu University in Japan.
The researchers showed that "germ-free" mice those that never came into contact with microbes - pumped out twice the amount of stress hormone when distressed than normal mice.

The animals were identical except for their microbes. It was a strong hint that the difference was a result of their micro-organisms.

"We all go back to that first paper for the first wave of neuroscientists considering microbes," says Dr Jane Foster, a neuropsychiatrist at McMaster University in Canada.

"That really was very powerful for those of us who were studying depression and anxiety."

It was the first hint of microbial medicine in mental health...and most microbes enter the body through the mouth. Dental hygiene is important.

Citation: Samuel A. Nigro. Dental Self Treatment--Universal Design: To Prevent Caries and Extractions (Updated 2018). ARC Journal of Dental science. 2018; 3(1): 3-5. doi:dx.doi.org/ 10.20431/2456-0030. 0301002 .

Copyright: (c) 2018 Authors. This is an open-access article distributed under the terms of the Creative Commons Attribution License, which permits unrestricted use, distribution, and reproduction in any medium, provided the original author and source are credited. 